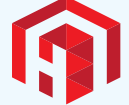 Heighten Science \\ P U B L I C I T I O N S Corporation \\ ISSN \\ 2575-0143}

\title{
Single-centre real world experience of the Mynx Femoral closure device in patients undergoing percutaneous coronary intervention
}

\author{
Thirunavukarasu $\mathbf{S}^{1}$, Zaman $\mathbf{M}^{1}$, Hayat $\mathbf{A}^{1}$, Aghamohammadzadeh \\ $\mathbf{R}^{1}$ and Malik $\mathbf{N}^{1,2 *}$
}

'Manchester Heart Centre, Purple Zone 1st Floor, Manchester University, NHS Foundation Trust, Oxford Road, Manchester M13 9WL, UK

${ }^{2}$ The University of Manchester, Room 3.31b, Core Technology Facility, 46 Grafton Street, Manchester M13 9NT, UK

*Address for Correspondence: Nadim Malik Manchester Heart Centre, Purple zone 1st Floor, Manchester University, NHS Foundation Trust, Oxford Road, Manchester M13 9WL, UK.

The University of Manchester, Room 3.31b, Core Technology Facility, 46 Grafton Street, Manchester M13 9NT, UK, Tel: 0044161

2768907; Email: nadim.malik@manchester.ac.uk

\section{MANCHESTER}

Submitted: 22 May 2019

Approved: 03 June 2019

Published: 04 June 2019

Copyright: () 2019 Thirunavukarasu S, et al. This is an open access article distributed under the Creative Commons Attribution License, which permits unrestricted use, distribution, and reproduction in any medium, provided the original work is properly cited

Keywords: Vascular closure device; Mynx; Percutaneous coronary interventions

Abbreviations: ACS: Acute Coronary Syndrome; BP: Blood Pressure; FDA: Food and Drug Administration; $\mathrm{PCl}$ : Percutaneous Coronary Interventions; PPCl: Primary PCl; PEG: Poly Ethylene Glycol; VCD: Vascular Closure Device

Check for updates

\section{Abstract}

Background: Vascular closure devices are routinely used following femoral artery access to perform percutaneous coronary interventions $(\mathrm{PCl})$. A number of devices are available on the market. We have reported previously on our experience of the Mynx device following diagnostic coronary angiography.

Aims: To assess the success and complication rates of the Mynx device used in all-comers in the context of $\mathrm{PCl}$ within a single cardiac centre.

Methods: Retrospective analysis of data available for patients who underwent PCl via the femoral route and received a Mynx device at a single tertiary centre.

Results: The device was used to achieve haemostasis in 113 patients following $\mathrm{PCl}$. In all cases weight-adjusted Heparin as well as dual antiplatelet therapy (Aspirin and Clopidogrel/ Ticagrelor) was administered as per $\mathrm{PCl}$ protocol. The device was successfully deployed in 111 cases (98.2\%). The were 2 cases of device failure, one due to operator error and the other due to Mynx grip balloon bursting during device deployment. In 15 cases (13.2\%; 9 male and 6 female) there were reports of small haematomas $(<2 \mathrm{~cm})$ or oozing resulting in application of manual pressure or Femstop (St. Jude). A further patient required ultrasound-guided compression of the artery due to a large retroperitoneal bleed resulting from access complications. A larger proportion of the cases with complications were done as $\mathrm{PPCl}(44 \%$ vs $33 \%)$. The group with complications had higher systolic BP $(140 \mathrm{mmHg}$ vs $128 \mathrm{mmHg} ; \mathrm{P}<0.05)$ and MABP $(97 \mathrm{mmHg}$ vs $75 \mathrm{mmHg} ; \mathrm{P}<0.05)$ as compared with the group without any complications.

Conclusion: The Mynx closure device is safe and easy to use in the context of $\mathrm{PCl}$, in both the elective and emergency (PPCl) settings. Complication rates, predominately minor, can be minimised in experienced hands.

\section{Introduction}

Large-bore artery haemostasis has been one of the main challenges in cardiology since the advent of percutaneous coronary interventions. Whilst the Radial artery approach to PCI has become increasingly common in most UK centres, the growing popularity of procedures requiring large-bore Femoral access, such as complex PCI, Balloon Aortic Valvuloplasty (BAV), Transcatheter Aortic Valve Implantation (TAVI) and the use of devices such as Micra (leadless pacemaker) and Impella (ventricular support device) has reignited discussions on the best means to achieve suitable haemostasis and minimising complications such as haematomas and life-threatening bleeds. Vascular closure devices are in frequent use for achieving femoral haemostasis 
and techniques such as the application of manual pressure or use of devices such as Femstop (St. Jude) are mostly used as a bailout strategy. This remains the case even after failure to achieve adequate haemostasis using any of the specific devices such as Mynx, Preclose or Angioseal.

An ideal arteriotomy closure device should avoid the use of permanent implants, be simple to use with predictable outcomes regardless of operator skill level, minimise trauma, have minimal effect on platelet activation as well as infections and successfully achieve haemostasis despite use of antiplatelets and anticoagulants. Data suggests that the Mynx device satisfies all these criteria.

The Mynx device gained FDA approval in 2010 and is unique in its delivery of a polyethylene glycol (PEG) gel to the surface of the artery without any mechanical anchoring [1], thereby categorised as a passive closure device. PEG, a bio-inert polymer with a good safety profile is a synthetic biocompatible material and has been used extensively in medical devices. The Mynx grip PEG sealant encompasses 2 materials: a 14.5-mm porous matrix and a 1.5-mm distal tip with grip technology [2]. The porous structure of the PEG allows it to absorb blood and subcutaneous fluids allowing it to rapidly expand to three to four times its original dimensions and filling the entire tissue tract. This can often manifest as a small swelling/hematoma at the site of implantation. After haemostasis is achieved, the sealant begins a process of hydrolysis, dissipating within 30 days [3]. Mynx devices achieve haemostasis without cinching, clamping or suturing which helps to provide a gentle closure for the patient. PEG is not known to interact with platelets or immune cells, reducing intravascular and inflammatory complications [2].

In a recent study of 4074 patients, Baker et al., have reported comparable safety and efficacy outcomes between Angioseal, which is the most commonly used active anchoring vascular closure device (VCD), and the passively deployed Mynx. The Mynx closure device remains desirable as there is no intra-arterial anchor upon device removal [4]. Other studies have reported similar rates of major vascular complications in patients who have received Angioseal versus Mynx following PCI with dual antiplatelets and LMW Heparin administered during the procedure [5].

We have reported previously that Mynx can be used safely and reliably in the setting of diagnostic coronary angiography [6]. In this study, we aimed to report the safety and efficacy of the Mynx closure device from real world data in patients undergoing interventional procedures at a single tertiary centre and factors contributing to all levels of risks involved. It is important to note that patients undergoing PCI have dualantiplatelet therapy as well as peri-procedural Heparin and not uncommonly IIb/IIIa inhibitors, thus increasing the chance of haematoma formation.

\section{Methods}

Patients who underwent PCI via the femoral artery followed by arterial closure using the Mynx device in a single tertiary centre were included in this study. All procedures were performed at Manchester Royal Infirmary, Manchester, UK. Demographic data as well as the use of antiplatelets/anticoagulation, baseline renal function, body mass index $>25$, past medical history and comorbidities was recorded for analysis. Positive history of hypertension was defined as patients already treated with antihypertensive drugs or previous systolic blood pressure (BP) $\geq 160 \mathrm{~mm} \mathrm{Hg}$ or diastolic BP $\geq 95 \mathrm{~mm}$ $\mathrm{Hg}$. Positive history of hypercholesterolemia was defined as total serum cholesterol $\geq 5 \mathrm{mmol} / \mathrm{L}$. Diagnosis of diabetes mellitus was defined as need for glucose-lowering medications, or previous fasting glucose $\geq 11.1 \mathrm{mmol} / \mathrm{L}$, or diagnostic response to the oral glucose tolerance test. Active smoking was defined as smoking within the last 3 months. In addition, procedural data including sheath size, and indication for PCI were divided into elective, ACS and PPCI were recorded. Further comparison was made 
between the group with any reported complication and those without; with the aim of identifying any predisposing factors.

Femoral artery access was achieved using a standard Seldinger technique under aseptic conditions. Intra-arterial BP was recorded prior to sheath removal. In the minority of cases where data on intra-arterial BP were not available, bedside BP measured using standard sphygmomanometer was noted prior to the procedure. Aortic systolic and diastolic blood pressures were recorded from the arterial pressure trace prior to sheath removal. Mean arterial BP (MABP) was estimated as: diastolic BP $+1 / 3 \times$ (systolic BP - diastolic BP).

Elective patients undergoing PCI and taking warfarin stopped this 5 days prior to the procedure and proceeded with PCI only if international normalized ratio was $<1.5$ on the day of the procedure. Patients taking dual-antiplatelet therapy continued on their regular medication. Post Mynx deployment care was as per manufacturer recommendations. Briefly, following Mynx device deployment, patients were placed on bedrest for 30 minutes, after which a wound inspection was carried out; in the absence of any signs of bleeding/ haematoma, patients could ambulate. All access-site complications (hematoma $<2 \mathrm{~cm} / 2-5 \mathrm{~cm} />5 \mathrm{~cm}$ ), bleeding, pseudoaneurysm) were documented in addition to device failure and conversion to manual compression or FemStop prior to patient ambulation/discharge.

Mynx efficacy was defined as the ability of the device to achieve full hemostasis. Device failure was defined as failure to stop bleeding after device deployment and conversion to manual compression or FemStop. Safety of the device was determined by rate of complications; bleeding was defined as any hemoglobin reduction requiring transfusion or requiring surgical intervention, and significant hematoma was defined as $>5 \mathrm{~cm}$ diameter [7].

\section{Statistical analysis}

Graph Pad Prism was used for data analysis and appropriate t-tests were applied according to categorical or continuous data. For all analyses, the criterion for statistical significance was set at $P<.05$.

\section{Results}

113 patients underwent closure of their femoral artery with the Mynx device (58\% male, $42 \%$ female). 18 experienced complications including 2 cases of unsuccessful device deployment, one due to operator error and second due to the Mynx grip balloon bursting during deployment. There was one case of major retroperitoneal haemorrhage due to access complications and requiring ultrasound-guided application of Femstop. In 15 cases (13.2\%), manual pressure in the form of Femstop (St Jude) was applied after development of a small haematoma measuring $<5 \mathrm{~cm}$ (Figure 1). There was no incidence of a large haematoma $(5 \mathrm{~cm})$, infection, distal embolization, pseudoaneurysms or presentations due to pain or discomfort following the use of the Mynx device.

To investigate the factors contributing to device challenge and complications, we compared demographic, procedure-related and clinical settings between the 2 groups; complication versus no complication. Results are shown in tables 1,2 .

The group associated with complications $(\mathrm{n}=18)$ had higher systolic BP $(140 \mathrm{mmHg}$ vs $128 \mathrm{mmHg} ; \mathrm{P}<0.05)$ and MABP $(97 \mathrm{mmHg}$ vs $75 \mathrm{mmHg} ; \mathrm{P}<0.05)$ compared with the group without any complications $(\mathrm{n}=95)$. Current smoking was associated with increased incidence of complications ( $45 \%$ vs $30 \%$ ) and statin use was frequent in the group without complications (67.7\%) as compared with the complications group (40\%). 


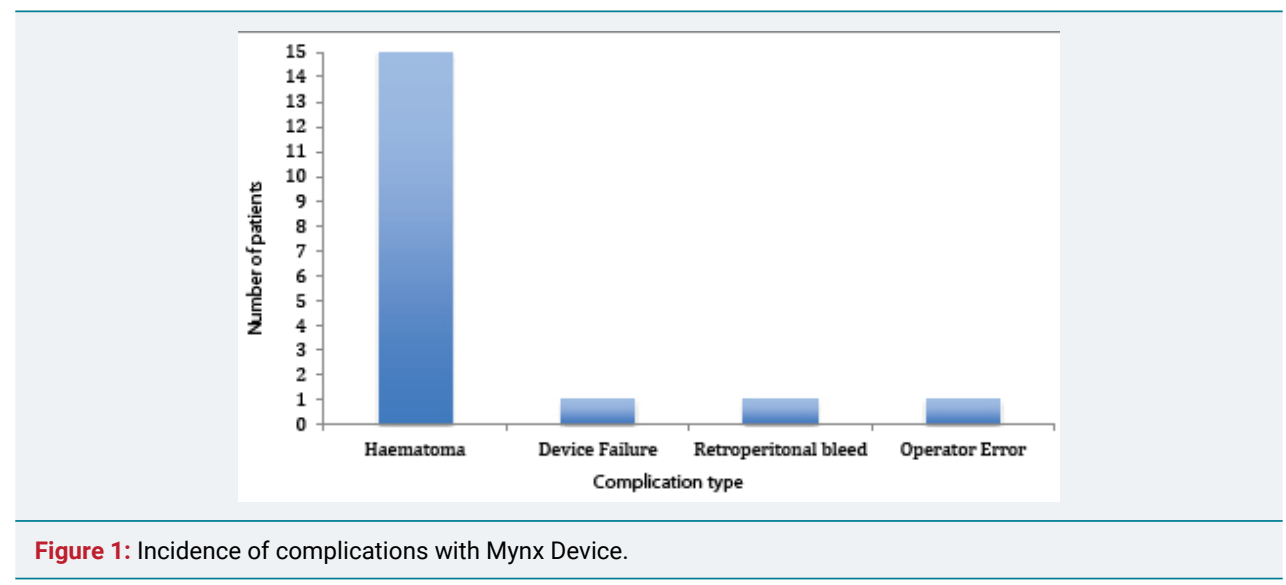

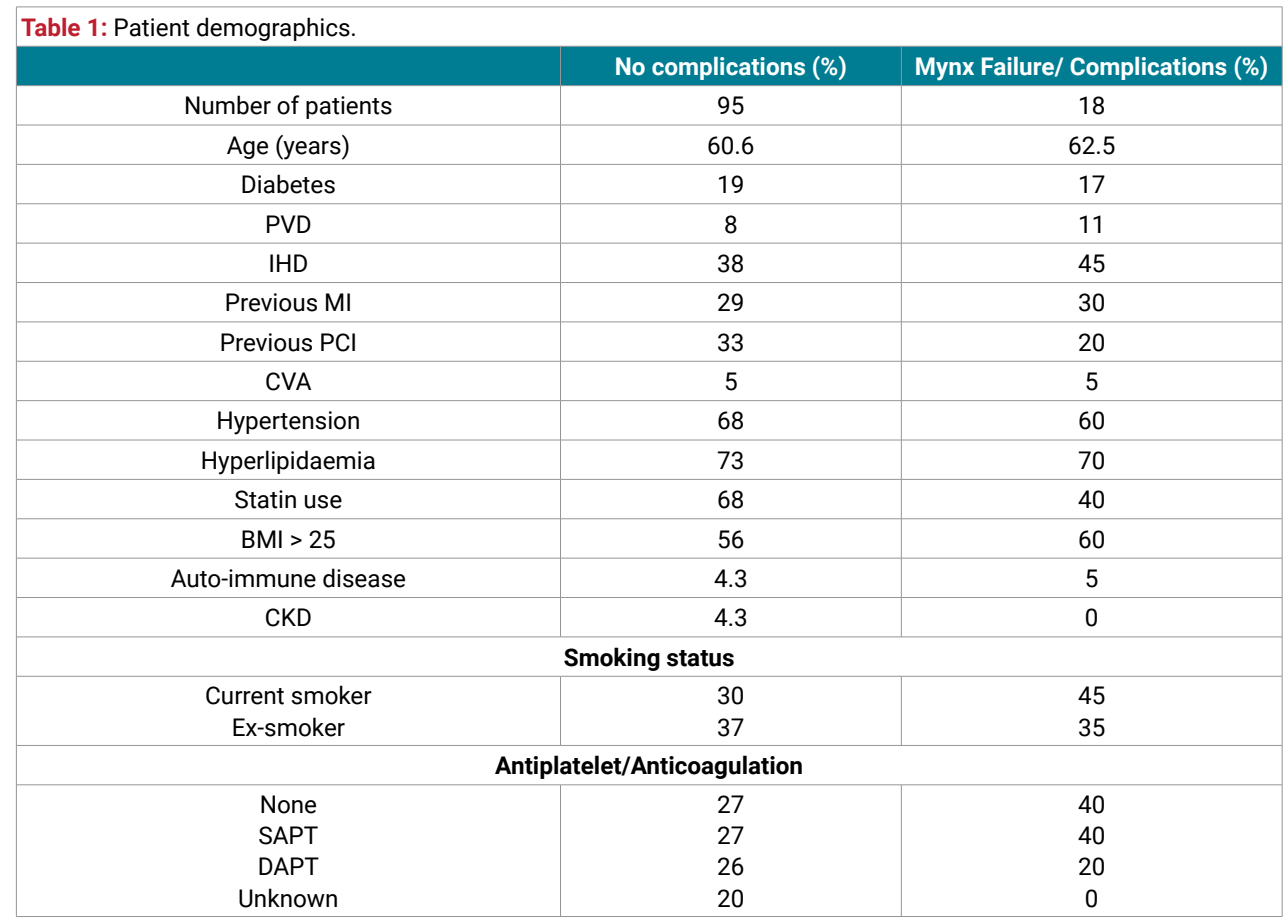

Table 2: Comparison of procedure related factors.

\begin{tabular}{|c|c|c|}
\hline & No complications (\%) & Mynx failure/ complication (\%) \\
\hline Number of patients & 95 & 18 \\
\hline \multicolumn{3}{|c|}{ Indication for intervention } \\
\hline Elective & 37 & 28 \\
\hline $\mathrm{PPCl}$ & 33 & 44 \\
\hline \multicolumn{3}{|c|}{ Sheath size } \\
\hline $6 \mathrm{~F}$ & 89 & 100 \\
\hline $7 F$ & 11 & 0 \\
\hline \multicolumn{3}{|c|}{ Periprocedural BP $(\mathrm{mmHg})$} \\
\hline Systolic & $128 *$ & $140 *$ \\
\hline Diastolic & 71 & 76 \\
\hline MABP & $75^{\star}$ & $97 *$ \\
\hline
\end{tabular}

The group associated with complications had a higher proportion of PPCI (44\%) procedures as compared with the group without complications (33\%).

\section{Discussion}

Closure with the Mynx device leads to enhanced patient comfort, rapid haemostasis, reduced time to ambulation, and hospital stay $[8,9]$. Published results with Mynx are variable with some studies reporting it is safe and effective and others reporting less favourable complication rates mainly in the early stages of deployment and especially associated with a new operator's learning curve [7-10]. 
Our study found that the Mynx was indeed an effective closure device $(98.9 \%$ successful deployment). 18 (15.9\%) patients encountered some device-related complications. From the subset of patients with associated complications, $83 \%$ were documented as having small haematomas $(<5 \mathrm{~cm})$ and were managed conservatively using pressure application. Only one patient suffered a major complication which was due to a large retroperitonal haemorrhage due to difficult access following a PPCI procedure. PPCI cases were associated with a higher incidence of haematomas $(<2 \mathrm{~cm})$. This may be attributed to the high initial/loading doses of dual antiplatelet therapy administered as per agreed protocols before PCI in addition to the peri-procedural heparin. However, the study as mentioned above demonstrated at least $90.8 \%$ success rate using the Mynx closure device in the patients on dual antiplatelet therapy with therapeutic heparin dose achieved.

The incidence of haematoma-related complications observed in our study appears to be higher than in other studies. This is because in our study any haematoma is counted as a complication. In reality, the formation of a small haematoma $(<2 \mathrm{~cm})$ is part of the process of deploying a Mynx and allows the PEG plug to expand to its full potential. Some of the complication rate could be also attributable to centre/operator level experience in using the Mynx device, a finding that has been previously associated with other vascular closure device complications [11].

We observed that peri-procedural BP recordings were higher in the 'complication group'. Studies have reported that patients undergoing PCI with higher systolic blood pressures are significantly more likely to have vascular access-related complications than patients with lower blood pressures [12,13]. Unlike a previous small study [10], we did not encounter any incidence of pseudoaneurysms, requirement for surgical repair or intravascular placement of the sealant although no follow up vascular imaging was performed as a matter of routine. Previous comparison of Mynx with other vascular closure devices such as AngioSeal have not shown a significant difference in major vascular complications, however minor complications such as device failure requiring $>30$ minutes of manual compression after device deployment occurred more in the Mynx (9.2\%) patient group versus Angioseal (3.7\%) [5]. The Mynx device has been shown to have a lower surgical repair rate than both Angioseal and Manual compression [14].

\section{Conclusion}

The Mynx device is a safe and effective femoral arteriotomy closure device in patients undergoing percutaneous coronary interventions (PCI). The complications associated with the Mynx are predominately minor. The complication rate may be reduced by operator experience, optimizing blood pressure control before and after the procedure, and ensuring femoral access is obtained at the correct anatomical landmark with screening. This device offers great patient comfort and is of great value even in high-risk subjects (bifurcation at site of puncture, calcified artery or dissection of femoral artery).

\section{Study Limitations}

Our study is a retrospective analysis of Mynx femoral arteriotomy closure in a single centre. The number of patients analyzed is small and therefore the study may be underpowered. There was no routine imaging of the puncture site post procedure. Most complications were identified on the same day as the procedure. There was no formal follow up although patients were asked to contact the study coordinator if there were signs of any bruising, bleeding or swelling hence there was a potential for some patients being lost to follow up.

\section{References}

1. Resnic FS, Majithia A, Marinac-Dabic D, Robbins S, Ssemaganda H, et al. Registry-Based Prospective, Active Surveillance of Medical-Device Safety. N Engl J Med. 2017; 376: 526-535. Ref.: https://bit.ly/2Kp4Eqi 
2. Grandhi R, Kanaan H, Shah A, Harrison G, Bonfield C, et al. Safety and efficacy of percutaneous femoral artery access followed by Mynx closure in cerebral neurovascular procedures: a single center analysis. J Neurointerv Surg. 2014; 6: 445-450. Ref.: https://bit.ly/2WBabAz

3. Taha A, Walsh EK, Wright KA, Ahmed I, Supakul N, et al. Safety and feasibility of a novel vascular closure device in neurointerventional procedures. Interv Neuroradiol. 2013; 19: 353-358. Ref.: https://bit.ly/2wyaX2v

4. Baker NC, Escarcega RO, Lipinski MJ, Magalhaes MA, Koifman E, et al. Active Versus Passive Anchoring Vascular Closure Devices Following Percutaneous Coronary Intervention: A Safety and Efficacy Comparative Analysis. J Interv Cardiol. 2016; 29: 108-112. Ref.: https://bit.ly/2WIFXIK

5. Azmoon S, Pucillo AL, Aronow WS, Ebrahimi R, Vozzolo J, et al. Vascular complications after percutaneous coronary intervention following hemostasis with the Mynx vascular closure device versus the AngioSeal vascular closure device. J Invasive Cardiol. 2010; 22: 175-178. Ref.: https://bit.ly/2wFhOXP

6. Hutchings D, Hayat A, Karunakaran A, Malik N. Success, Safety, and Efficacy of the Mynx Femoral Closure Device in a Real-World Cohort: Single-Center Experience. J Invasive Cardiol. 2016; 28: 104108. Ref.: https://bit.ly/2HQYyNv

7. Islam MA, George AK, Norris M. Popliteal artery embolization with the Mynx closure device. Catheter Cardiovasc Interv. 2010; 75: 35-37. Ref.: https://bit.ly/2HRVReG

8. Fargen $\mathrm{KM}, \mathrm{Hoh} \mathrm{BL}$, Mocco J. A prospective randomized single-blind trial of patient comfort following vessel closure: extravascular synthetic sealant closure provides less pain than a self-tightening suture vascular compression device. J Neurointerv Surg. 2011; 3: 219-223. Ref.: https://bit.ly/2QHJ7Ki

9. Scheinert D, Sievert H, Turco MA, Schmidt A, Hauptmann KE, Mueller R, et al. The safety and efficacy of an extravascular, water-soluble sealant for vascular closure: initial clinical results for Mynx. Catheter Cardiovasc Interv. 2007; 70: 627-633. Ref.: https://bit.ly/2wBotIT

10. Fields JD, Liu KC, Lee DS, Gonda SJ, Dogan A, et al. Femoral artery complications associated with the Mynx closure device. AJNR Am J Neuroradiol. 2010; 31: 1737-1740. Ref.: https://bit.ly/311JYue

11. Resnic FS, Wang TY, Arora N, Vidi V, Dai D, et al. Quantifying the learning curve in the use of a novel vascular closure device: an analysis of the NCDR (National Cardiovascular Data Registry) CathPCI registry. JACC Cardiovasc Interv. 2012; 5: 82-89. Ref.: https://bit.ly/2wybLo3

12. Manoukian SV, Feit F, Mehran R, Voeltz MD, Ebrahimi R, et al. Impact of major bleeding on 30-day mortality and clinical outcomes in patients with acute coronary syndromes: an analysis from the ACUITY Trial. J Am Coll Cardiol. 2007; 49: 1362-1368. Ref.: https://bit.ly/2ESgQMG

13. Sulzbach-Hoke LM, Ratcliffe SJ, Kimmel SE, Kolansky DM, Polomano R. Predictors of complications following sheath removal with percutaneous coronary intervention. J Cardiovasc Nurs. 2010; 25: E1-8. Ref.: https://bit.ly/2HSKa7x

14. Noor S, Meyers S, Curl R. Successful reduction of surgeries secondary to arterial access site complications: a retrospective review at a single center with an extravascular closure device. Vasc Endovascular Surg. 2010; 44: 345-349. Ref.: https://bit.ly/2XmocPx 